\title{
WILEY-VCH
}

DOI: 10.1002/((please add manuscript number))

Article type: Communication

\section{Hydrophobic Light-to-Heat Conversion Membranes with Self-Healing Ability for Interfacial Solar Heating}

Lianbin Zhang, Bo Tang, Jinbo Wu, Renyuan Li, and Peng Wang*

Dr. L. B. Zhang, B. Tang, Dr. J. B. Wu, R.Y. Li, Prof. P. Wang

Water Desalination and Reuse Center, Division of Biological and Environmental Sciences and Engineering, King Abdullah University of Science and Technology, Thuwal 23955-6900, Saudi Arabia.

E-mail: peng.wang@kaust.edu.sa

Keywords: light-to-heat conversion, self-healing, wetting, interfacial heating, membrane.

Water evaporation is an endothermic process, in which heat is absorbed. In nature, water evaporation is an important part of the global water cycle and is driven by solar radiation as heat source, which is abundantly and freely available. ${ }^{[1,2]}$ Generally, the solar irradiation penetrates water through a certain depth depending on water quality and the spectrum wavelength, and the water profile over the light passage length is heated up homogeneously. Over the history of mankind, this natural water evaporation has been widely imitated at various scales for beneficial uses. ${ }^{[3-7]}$ For example, in some remote and rural areas where access to centralized drinking water supply is unavailable, solar distillation is used to produce freshwater, which uses solar energy to heat and evaporate seawater or brackish water. ${ }^{[5-7]}$ However, the nature's way of water evaporation is imperfect, and it falls into the category of bulk heating, in which the entirety of bulk water is uniformly heated up. One fact that is often overlooked is that water evaporation is indeed a surface process, in which water molecules at the very thin air-water interface in the liquid water side, driven by their high energy state, are transported into the vapor phase. Thus, the conventional bulk heating of water is not a rational choice for water evaporation and it would unavoidably results in unnecessary heat/energy loss due to the energy transfer to the non-evaporative portion of the bulk water. It is thus not 


\section{WILEY-VCH}

surprising that a major problem in the solar distillation and other solar evaporation processes is their low energy efficiency, similar to that in the natural water cycle. ${ }^{[6,7]}$

Therefore, targeting at enhancing only the local temperature of interfacial water is more meaningful and energy-efficient for a high evaporation rate. The localized heating at the interfacial water can generate a sharper temperature gradient with the same level of energy input. In comparison to uniformly high temperature profile in the bulk water heating scheme, the localized high temperature of the interfacial water in the interfacial heating scheme minimizes the heat loss by the non-evaporative lower part of the bulk water due to its high temperature. Based on this understanding, it is thus logical to gather solar radiation only at the water-air interface so to generate localized and concentrated high temperature of the interfacial water. Recent efforts in this regard are embodied exclusively by inorganic light-toheat conversion (photothermal) materials, ${ }^{[8-11]}$ including carbon-based or noble metal nanoparticle materials with proper hydrophobicity and these photothermal materials absorb light and covert it to heat energy. ${ }^{[8-18]}$ The surface hydrophobicity, given generally by alkyl or fluoroalkyl group surface modification, is a prerequisite to a self-floating capability of these materials at air-water interface, which in turn permits an automated and uninterrupted interfacial solar heating.

However, in practical application, chemical oxidation from exposure to highly oxidative chemicals in water and air (e.g., hypochlorites, ozone), strong light (e.g., ultraviolet light), etc. results in deterioration of surface hydrophobicity due to the gradual decomposition or removal of these hydrophobic organic functional groups (e.g., alkyl or fluoroalkyl), leading to a transition from a hydrophobic to a hydrophilic wettability of the materials. ${ }^{[19-29]}$ With a hydrophilic wettability, the photothermal materials loss their capability of self-sustained interfacial heating. As a result, a hydrophobic photothermal material which can heal and recover its hydrophobicity autonomously upon degradation, namely hydrophobicity selfhealing ability, can be a judicious solution to lead to elongated applications of these materials. 


\section{WILEY-VCH}

While there has been very little literature report on the self-healing of hydrophobicity on inorganic materials, ${ }^{[23,24]}$ the hydrophobicity self-healing on polymeric materials have been widely studied and reported due to the polymers' dynamical behavior and easy chemical and mechanical tunability. ${ }^{[21,22,25-29]}$ With the wide availability of the knowledge of the hydrophobicity self-healing on polymeric materials, we believe that a careful selection of polymeric photothermal material would lead us to a highly efficient interfacial heating material with a hydrophobicity self-healing capability that promises long-term application of such material.

In this Communication, we report a proof-of-concept of the hydrophobicity self-healing photothermal membrane for the interfacial solar heating based on polypyrrole (PPy) coated stainless steel (SS) mesh. The membrane was prepared by deposition of PPy, a polymeric photothermal material, onto SS mesh substrate, followed by fluoroalkylsilane modification of the PPy coating to achieve desirable hydrophobicity. The surface wettability of membrane was controlled to conform the Wenzel's wetting behavior, which enabled the membrane to spontaneously stay at the water-air interface and allowed for sufficient contact with the water surface so to precisely heat the interfacial water under the solar irradiation. PPy was rationally chosen as the photothermal polymer because of its broad spectrum absorption, high photothermal conversion efficiency, and its facile solution-based fabrication, which permits a uniform film coating with an easy control over the film thickness. ${ }^{[16-18]}$ From the self-healing perspective, the PPy coating film, once formed, can act as a matrix to preserve a large number of reacted fluoroalkylsilane moieties, which are the hydrophobicity self-healing agents in our case and can migrate to the PPy coating surface to restore the hydrophobicity upon the surface hydrophobicity loss. Our results showed that with the such-designed hydrophobic photothermal membrane floating on water surface, water evaporation rate could be significantly enhanced under otherwise the same conditions. We showed that upon the damage of the outmost fluoroalkylsilane functional groups on the membrane, the surface 


\section{WILEY-VCH}

could autonomously restore its hydrophobicity and the self-healing ability could also be accelerated with the solar light irradiation. Furthermore, the interfacial heating material in the form of membrane also benefits its operation and integration into water distillation device for practical applications compared with the powder-form materials, ${ }^{[8-10]}$ and in this study, a prototype and all-in-one solar distillation device was prepared to demonstrate the applicability of the concept of interfacial heating to point-of-use portable water production.

Our fabrication strategy of the self-healing hydrophobic photothermal membranes for the interfacial water heating is schematically shown in Figure 1a. The PPy coating was deposited onto the meshes by electropolymerization of pyrrole. The choice of the stainless steel (SS) meshes here as the substrate material is due to its inherent porous structure, its good mechanical and chemical stability, as well as its easy availability and low cost. Figure 1b shows the scanning electronic microscopic (SEM) images of the original unmodified SS mesh (500 mesh size) and the mesh deposited with PPy by electropolymerization for 1 hour. The original mesh with 500 mesh size has an average pore size of 26.8 $\mu \mathrm{m}$ (Figure 1b1) and smooth wire surface, with knitting wire diameter of $24.5 \mu \mathrm{m}$. After the deposition of PPy, a PPy coating with micro-sized surface structures could be clearly seen (Figure 1b2 and Supporting Information Figure S1). It has been found that the thickness of the PPy coating on the wires increased almost linearly with the electropolymerization time, with the coating thickness being 2.6 $\mu \mathrm{m}$ at 1-hour electropolymerization (Figure 1c).

To achieve the self-floating capability, the PPy-coated meshes were then treated by chemical vapor deposition (CVD) of fluoroalkylsilane to reduce their surface energy. ${ }^{[21,30]}$ As shown in Figure 1d, after the hydrophobic modification the meshes exhibited high water contact angles, which increased gradually with the electropolymerization time in the PPy deposition step. When the PPy deposition time exceeded $30 \mathrm{~min}$, the contact angle reached a constant value of $\sim 140^{\circ}$. Furthermore, the meshes, although highly hydrophobic, exhibited high adhesion to water droplets. As shown in the insets of Figure 1d, for the PPy-coated mesh prepared by 1- 


\section{WILEY-VCH}

hour electropolymerization and fluoroalkylsilane modification, when the mesh was placed perpendicular and even inverted up-side down, the water droplets still firmly adhered to the surface. This wettability with high water contact angle as well as high adhesion to water droplets of the hydrophobic meshes is regarded as Wenzel's wetting behavior, ${ }^{[30-32]}$ under which, although substrate surface exhibits a high contact angle, water is able to penetrate into the rough surface structures of the substrate and displace the otherwise air pockets present under Cassie's wetting. ${ }^{[32,33]}$ This Wenzel's wetting behavior of the meshes is a result of the combination of both the micro-sized surface structure and the hydrophobic modification. Under the Wenzel's wetting state, contact between the water and solid substrate surface is intimate and maximized, which is beneficial for the heat transfer from the mesh to water as air otherwise is considered as a poor heat conductor. A comparison of water evaporation rate between a Wenzel's and Cassie's wettability can be found in the Supporting Information (Figure S2), which clearly demonstrates that significantly higher water evaporation is achieved under the Wenzel's wetting state than the Cassie’s state under the otherwise same conditions.

Given the porous structure of the mesh substrate, a direct measurement of the light adsorption property of the PPy coating on the mesh deems impossible. Instead, to confirm the strong light absorption of PPy coating, a PPy coating was deposited onto an ITO glass substrate by electropolymerization method, followed by fluoroalkylsilane modification and the thusprepared coating exhibited a broad absorption band, extending from the visible to the near infrared (NIR) region with two pronounced peaks at $450 \mathrm{~nm}$ and $850 \mathrm{~nm}$, with the absorbance increasing with the thickness of the PPy coating. The broad band adsorption is characteristic of the bipolaronic metallic state of the doped polypyrrole (see Supporting Information Figure

S3a). ${ }^{[16-18]}$ It is worth pointing out that (1) when the thickness of the PPy coatings were greater than $1.2 \mu$ m (i.e., with PPy deposition time $>15 \mathrm{~min}$ ), the absorbance of the PPy coatings saturated the limitation of the current UV-vis-NIR spectrophotometer; (2) the PPy coatings 


\section{WILEY-VCH}

on the ITO glass showed negligible reflection and transmission (Figure S3b and 3c); (3) the PPy coatings with and without fluoroalkylsilane modification exhibited almost the same light absorption spectra, indicating negligible influence of the fluoroalkylsilane modification on the photothermal property of the PPy coatings. All the above results demonstrated the strong light absorbance by the PPy coating.

The photothermal property of the PPy-coated meshes was then investigated. A simulated solar light with an intensity of $1000 \mathrm{Wm}^{-2}$ was shined on the PPy-coated meshes and an FLIR infrared camera was used to probe the temperature of the meshes in air. Under the light irradiation, a steady-state temperature for the meshes with the PPy coating could be reached, which is defined as the temperature when the heat generation under light irradiation and heat dissipation due to radiative heat flux is at equilibrium. Figure 2a shows the steady-state temperature of the PPy-coated SS meshes as a function of the coating thickness. As can be seen, with the ambient temperature being constant at $\sim 22^{\circ} \mathrm{C}$, the mesh temperature first increased steadily with the coating thickness before reaching a plateau (i.e., $\sim 55^{\circ} \mathrm{C}$ ) when the PPy coating thickness was beyond $1.6 \mu \mathrm{m}$. Figure $2 \mathrm{~b}$ presents the kinetics of temperature under light irradiation. Upon the simulated solar irradiation, the temperature of the PPycoated mesh increased quickly to its steady-state temperature of $53 \pm 2{ }^{\circ} \mathrm{C}$ while for the original unmodified mesh showed a steady-state temperature of $\sim 27^{\circ} \mathrm{C}$, which represented only $\sim 5{ }^{\circ} \mathrm{C}$ increased under the irradiation. It should be mentioned that because of its relatively low emissivity ( $\sim 16)$ of the original SS materials, the temperature of the unmodified mesh measured by the IR camera was a little lower than its actual temperature $\left(\sim 30^{\circ} \mathrm{C}\right.$ as measured by a thermocouple). This result clearly demonstrated the efficient lightto-heat conversion property of the mesh, which was imparted by the PPy coating. Having confirmed the photothermal effect, we then measured the water evaporation performance with the photothermal mesh membrane floating on water surface. Figure 3a presents the schematic setup for the water evaporation measurement. Briefly, the 


\section{WILEY-VCH}

photothermal membrane was placed and self-floated on water surface in a beaker, which was kept on an electronic balance to monitor the real-time water mass change. The same simulated solar light with an intensity of $1000 \mathrm{Wm}^{-2}$ was used and shined directly onto the self-floating membrane. As a control, in the absence of the membrane on the water surface, the water loss due to evaporation from the beaker with and without simulated solar light irradiation was 3.9 $\mathrm{kg} \mathrm{m}^{-2}$ and $0.88 \mathrm{~kg} \mathrm{~m}^{-2}$ for 10 hours, respectively. Due to its hydrophobicity, the PPy-coated SS mesh spontaneously stayed at the water-air interface, and moved along with water surface during evaporation, which allows for automated and targeted interfacial water heating under light irradiation. Figure 3b shows the mass of the water evaporated as a function of the irradiation time in the presence of the photothermal membranes. The amount of water evaporated increased first with increasing PPy coating thickness before reaching a plateau (i.e., $~ 9.2 \mathrm{~kg} \mathrm{~m}^{-2}$ ) when the PPy coating thickness was $1.6 \mu \mathrm{m}$, as shown in Figure 3c. Clearly, compared to the control case without the membrane, the water evaporation was greatly enhanced in the presence of membranes. For the mesh with the PPy coating thickness of 2.6 um, the water evaporation amounted to $9.2 \mathrm{~kg} \mathrm{~m}^{-2}$ for 10 hours, which represented $235 \%$ that of the case without the membrane. Also, for a bare SS mesh (i.e., without PPy coating) with hydrophobic modification, which was also able to self-float on water surface, it produced 5.4 $\mathrm{kg} \mathrm{m}^{-2}$ for 10 hours.

To probe more into the mechanism of the enhanced water evaporation by the self-floating photothermal membrane, IR thermal images were captured and the temperature profiles of the water were analyzed. As shown in Figure 3d, without the light irradiation, the water in both beakers with and without the membranes exhibited almost same homogeneous temperature profiles, and the temperature of the bulk water was both close to the room temperature of $\sim 22^{\circ} \mathrm{C}$. Upon the light irradiation, the interfacial water temperature in the presence of the membrane increased immediately (middle in Figure3d), with a steady-state temperature as high as $\sim 39^{\circ} \mathrm{C}$. Figure $3 \mathrm{f}$ compares the water surface temperature profiles of two beakers as a 


\section{WILEY-VCH}

function of the irradiation time and clearly an obvious enhancement was observed in the presence of the membrane. After $2 \mathrm{~h}$ light irradiation, the thermal image of the beaker with the surface self-floating membrane (the right panel of Figure 3d) shows a sharper temperature gradient than the one without the membrane (Figure 3e), where a homogeneous water temperature profile was obtained instead. The fact that, under solar irradiation, a uniform water temperature profile in the beaker in the absence of the membrane (the right panel in Figure 3c) was obtained provides strong evidence that the heating without the membrane falls into the conventional bulk heating scheme. The results confirm our strategy of interfacial heating.

The light energy to heat of water evaporation conversion efficiency $(\eta)$ of the photothermal membranes can be estimated by Equation (1):

$$
\eta=Q_{e} / Q_{s}
$$

Where $Q_{s}$ is the incidence light power $\left(1000 \mathrm{~W} \mathrm{~m}^{-2}\right)$, and $Q_{e}$ is the power of evaporation of the water, which can be estimated by Equation (2).

$$
Q_{e}=\frac{d m \times H_{e}}{d t}=v \times H_{e}
$$

where $m$ is the mass of evaporated water, $t$ is time, $v$ is the evaporation rate of water, and $H_{e}$ is the heat of evaporation of water ( $2260 \mathrm{~kJ} / \mathrm{kg}$ ). The water evaporation rate by the mesh with PPy coating thickness of $2.6 \mu \mathrm{m}$ (prepared by 1-hour electropolymerization) was then calculated to be $0.92 \mathrm{kgm}^{-2} \mathrm{~h}^{-1}$ and the conversion efficiency $(\eta)$ of the mesh was $\sim 58 \%$, which stands very favorably against the case without the membrane, whose conversion efficiency was only $24 \%$. This conversion efficiency of the photothermal membrane for the light energy to heat of water evaporation is also higher than that of most of the current solar stills, which exhibited typical efficiencies in the range of $30 \sim 45 \%{ }^{[7]}$

For the purpose of comparison, we also prepared a hydrophilic photothermal membrane of PPy-coated mesh and studied its water evaporation performance. First, it was found that once 


\section{WILEY-VCH}

the hydrophilic PPy-coated mesh contacted the water, water spread and penetrated the pores of the mesh, and the mesh quickly sunk to the bottom of the beaker. More importantly, with the hydrophilic photothermal membrane at the bottom of the water, a much lower water evaporation rate was observed (see Supporting Information Figure S4). The thermal image revealed that, instead of locally high temperature around the mesh, a homogenous temperature profile in the overlying water column was produced (Supporting Information Figure S5), which presumably was caused by the convection within the water column due to density variation. We also tested seawater evaporation performance by our photothermal membranes and similar level of performance was obtained (Supporting Information Figure S6), which promises the self-floating photothermal membranes a high potential toward water desalination. In this study, the mesh size is no trivial parameter as it determines pore size of the substrates and Supporting Information Figure S7 compares the water evaporation performance of the PPy-coated self-floating meshes with different sizes (80, 200, 325, and 500 mesh). It was found that the water evaporation rate increased with decreasing mesh pore size. The 500 mesh, which had the smallest pore size and thinnest wire diameter among these meshes, exhibited the highest evaporation rate. This is so because smaller pore size and wire diameter favors the deposited PPy to make more contact with the underlying water, and thus enhancing the conversion of light to heat.

To demonstrate the self-healing ability, the hydrophobic PPy-coated mesh (PPy coating thickness $\sim 2.6 \mu \mathrm{m}, 500$ mesh) was damaged artificially by a plasma treatment using air as gas source, which produces oxygen containing hydrophilic groups on the surface. ${ }^{[21-29]}$ After the treatment, the mesh surface became superhydrophilic with a water contact angle of $0^{\circ}$, which was attributed to the combination of the rough surface structure and the hydrophilic property of the oxygen-containing groups on the treated surface. However, when the plasmatreated mesh was stored under ambient condition $\left(\sim 22{ }^{\circ} \mathrm{C}\right.$ and relative humidity $\left.\sim 50 \%\right)$ for 6 hours, it gradually restored its hydrophobicity with water contact angle of $\sim 140^{\circ}$ at the end 


\section{WILEY-VCH}

(Figure 4a), indicating its hydrophobicity self-healing capability. This self-healing ability of the hydrophobic PPy-coated mesh surface can be attributed to the self-migration of the fluoroalkylsilane (POTS) within the PPy coating, which serves as a reservoir to accommodate an abundance of hydrophobic POTS molecules. ${ }^{[21-29]}$ Most importantly, we found that this self-healing ability of the hydrophobic PPy-coated mesh could be accelerated by solar light irradiation. As presented in Figure 4a, under the simulated solar light irradiation (1000 $\left.\mathrm{W} \mathrm{m}^{-2}\right)$, the restoration of the surface hydrophobicity was completed within 1 hour, in comparison to 6 hours under the ambient conditions. As demonstrated above, under the light irradiation, a considerably elevated temperature $\left(\sim 55^{\circ} \mathrm{C}\right)$ could be obtained on the PPy-coated mesh surface, which in turn promotes the migration and enrichment of the POTS molecules on the outmost mesh surface. The plasma-damaging/light-promoted healing process of the PPycoated mesh could be repeated for many times without significant reduction of the original hydrophobicity (Figure 4b). The self-healing capability of the hydrophobic photothermal membranes thus provides a promising way to enhance its durability for practical applications. The current strategy for the preparation of the self-healing hydrophobic photothermal membranes by electropolymerization of PPy onto SS mesh, followed with CVD of fluoroalkylsilane is suited for large-scale fabrication and has a great potential to be used for direct portable water production. In this study, a simple, and all-in-one solar distillation device was designed and fabricated as a prototype based on the concept of photothermal interfacial heating and the device is illustrated in Figure 5 (Supporting Information Figure S8). This device consists of light transparent plastic walls and two chambers (evaporation chamber and condensing chamber) connected in their upper parts but separated in their lower parts by a division. The evaporation chamber is bottomed with the hydrophobic photothermal membrane, which spontaneously floats on the surface of seawater or other type of source water. Under the nature solar light illumination, this chamber evaporates the seawater and guides the water vapor into the upper chamber, where a solar-powered electrical fan generates 


\section{WILEY-VCH}

an air flow field and drives the water vapor into a condensing chamber to collect freshwater.

The condensing chamber with the plastic bottom is in direct contact with the seawater and thus utilizes the seawater as a cold source to facilitate condensation. The results showed that the device with a $120 \mathrm{~cm}^{2}$ photothermal membrane $(10 \times 12 \mathrm{~cm})$, when placed on the water surface, an amount of $\sim 9.0$ g freshwater could be collected after 5-hour nature sunlight exposure. In a sharp contrast, the same device without the photothermal membrane collected only $\sim 0.6$ g water under the same sunlight exposure. The enhanced water collection capability by the device with the photothermal membrane floating on the water surface is the result of a significant increased water surface temperature in the evaporation chamber, which speeds up the evaporation, and the-thus resulted temperature differences between the two chambers continuously drives the water to transport into the condensing chamber. Furthermore, the photothermal membranes are quite stable, and their water evaporation performance did not show obvious change even after long-term exposure (over 100 hours) to simulated solar light or natural sunlight. This result demonstrates the practical application of photothermal membrane for point-of-use freshwater production.

In conclusion, we have demonstrated a self-healing hydrophobic photothermal membrane for interfacial solar heating, which involves accepting and converting solar light to heat only at water-air interface to generate a precisely local heating of interfacial water to enhance water evaporation. The concept was embodied for the first time by a rational integration of surface hydrophobicity and light-to-heat conversion material onto mesh-based membranes, which exhibited significantly enhanced water evaporation rates in comparison to conventional bulk heating scheme. Upon loss of their hydrophobicity, the membranes autonomously restored their hydrophobicity under ambient conditions and the restoration of the hydrophobicity could be accelerated under light irradiation. We believe that this study represents a new avenue for the design and fabrication of next-generation solar heating system and thus in the long term contributes to the global effort in addressing world water and energy issues. 


\section{WILEY-VCH}

\section{Experimental Section}

Materials. $\mathrm{HCl}$, and 1H,1H,2H,2H-perfluorooctyltriethoxysilane (POTS) were purchased from Sigma-Aldrich and pyrrole, and sodium dodecyl sulfate (SDS) were purchased from Acros. All these chemicals were used as received. Stainless steel (SS) meshes with different mesh sizes were purchased from Alfa Aesar (Karlsruhe, German). Deionized (DI) water purified in a Milli-Q (Millipore, Billerica, MA, USA) system was used in all experiments. Preparation of photothermal membranes. The SS meshes were cleaned with ethanol by sonication, rinsed with copious amount of ethanol and water, and dried with $\mathrm{N}_{2}$ flow. Polypyrrole (PPy) was then deposited on the cleaned mesh via an electropolymerization process of pyrrole. ${ }^{[34]}$ Briefly, the cleaned SS mesh and Pt electrode were immersed in an aqueous electrolyte solution containing $0.5 \mathrm{wt} \%$ SDS, $0.01 \mathrm{M} \mathrm{HCl}$, and $0.1 \mathrm{M}$ pyrrole. An electrical potential of $1.5 \mathrm{~V}$ was applied between the SS mesh (anode) and Pt electrode (cathode) using a Keithley 2400 power supply for certain period of time. After the deposition of PPy coating on the mesh surface, the meshes were rinsed with copious of water to remove SDS from the surface before drying under $\mathrm{N}_{2}$ flow. To reduce the surface energy, the PPycoated SS meshes were modified with a fluoroalkylsilane of POTS by chemical vapor deposition to obtain hydrophobic surfaces. ${ }^{[21,30]}$

\section{Supporting Information}

Supporting Information is available from the Wiley Online Library or from the author.

\section{Acknowledgements}

The authors thank KAUST Competitive Research Grants Program-Round 3 for funding.

Received: ((will be filled in by the editorial staff))

Revised: ((will be filled in by the editorial staff)) Published online: ((will be filled in by the editorial staff))

[1] T. Oki, S. Kanae, Science 2006, 313, 1068-1072.

[2] N. S. Lewis, Science 2007, 315, 798-801. 


\section{WILEY-VCH}

[3] A. Razmjou, Q. Liu, G. P. Simon, H. Wang, Environ. Sci. Technol. 2013, 47, 1316013166.

[4] V. Velmurugana, K. Srithar, Renew. Sustain. Energy Rev. 2008, 12, 2253-2263.

[5] R. D. Jackson, C. H. M. van Bavel, Science 1965, 17, 1377-1379.

[6] C. N. Li, Y. Goswami, E. Stefanakos, Renew. Sustain. Energy Rev. 2013, 19, 136-163.

[7] A. E. Kabeel, S. A. El-Agouz, Desalination 2011, 276, 1-12.

[8] H. Ghasemi, G. Ni, A. M. Marconnet, J. Loomis, S. Yerci, N. Miljkovic, G. Chen, Nat. Comm. 2014, 5, 4449.

[9] Y. Zeng, J. Yao, B. A. Horri, K. Wang, Y. Wu, D. Li, H. Wang, Energy Environ. Sci. 2011, 4, 4074-4078.

[10] Z. Wang, Y. Liu, P. Tao, Q. Shen, N. Yi, F. Zhang, Q. Liu, C. Song, D. Zhang, W. Shang, T. Deng, Small 2014, 10, 3234-3239.

[11] Y. Liu, S. Yu, R. Feng, A. Bernard, Y. Liu, Y. Zhang, H. Duan, W. Shang, P. Tao, C. Song, T. Deng, Adv. Mater. 2015, 27, 2768-2774.

[12] O. Neumann, A. S. Urban, J. Day, S. Lal, P. Nordlander, N. J. Halas, ACS Nano 2013, 7, 42-49.

[13] R. Jiang, S. Cheng, L. Shao, Q. Ruan, J. Wang, J. Phys. Chem. C 2013, 117, 8909-8915.

[14] E. Miyako, T. Deguchi, Y. Nakajima, M. Yudasaka, Y. Hagihara, M. Horie, M.

Shichiri, Y. Higuchi, F. Yamashita, M. Hashida, Y. Shigeri, Y. Yoshida, S. Iijima, Proc. Natl Acad. Sci. USA 2012, 109, 7523-7528.

[15] D. Jaque, L. M. Maestro, B. del Rosal, P. Haro-Gonzalez, A. Benayas, J. L. Plaza, E.

M. Rodríguez, J. García Solé, Nanoscale 2014, 6, 9494-9530.

[16] K. Yang, H. Xu, L. Cheng, C. Sun, J. Wang, Z. Liu, Adv. Mater. 2012, 24, 5586-5592.

[17] Z. Zha, X. Yue, Q. Ren, Z. Dai, Adv. Mater. 2013, 25, 777-782.

[18] M. Chen, X. Fang, S. Tang, N. Zheng, Chem. Commun. 2012, 48, 8934-8936. 


\section{WILEY-VCH}

[19] K. Uosaki, M. E. Quayum, S. Nihonyanagi, T. Kondo, Langmuir 2004, 20, 1207-1212.

[20] A. Siregar, M. Kleber, R. Mikutta, R. Jahn, European Journal of Soil Science 2005, 56, 481-490.

[21] Y. Li, L. Li, J. Q. Sun, Angew. Chem., Int. Ed. 2010, 49, 6129-6133.

[22] H. Wang, Y. Xue, J. Ding, L. Feng, X. Wang, T. Lin, Angew. Chem., Int. Ed. 2011, 50, $11433-11436$.

[23] X. Wang, X. Liu, F. Zhou, W. Liu, Chem. Commun. 2011, 47, 2324-2326.

[24] Q. Liu, X. Wang, B. Yu, F. Zhou, Q. Xue, Langmuir 2012, 28, 5845-5849.

[25] L. Ionov, A. Synytska, Phys. Chem. Chem. Phys. 2012, 14, 10497-10502.

[26] T. Dikić, W. Ming, R. A. T. M. van Benthem, A. C. C. Esteves, G. de With, Adv. Mater. 2012, 24, 3701-3704.

[27] H. Wang, H. Zhou, A. Gestos, J. Fang, H. Niu, J. Ding, T. Lin, Soft Matter 2013, 9, 277-282.

[28] Y. Li, S. Chen, M. Wu, J. Sun, Adv. Mater. 2014, 26, 3344-3348.

[29] S. Chen, X. Li, Y. Li, J. Sun, ACS Nano 2015, 9, 4070-4076.

[30] L. B. Zhang, J. B. Wu, M. Hedhili, X. L. Yang, P. Wang, J. Mater. Chem. A 2015, 3, 2844-2852.

[31] R. N. Wenzel, Ind. Eng. Chem. 1936, 28, 988-994.

[32] S. Wang, L. Jiang, Adv. Mater. 2007, 19, 3423-3424.

[33] A. B. D. Cassie, S. Baxter, Trans. Faraday Soc. 1944, 40, 546-551.

[34] T. An, S. J. Cho, W. S. Choi, J. H. Kim, S. T. Lim, G. Lim, Soft Matter 2011, 7, 98679870. 


\section{WILEY-VCH}

Figure 1. Preparation of the interfacial heating membrane on stainless steel mesh. (a) Schematic illustration for the preparation of the light-to-heat conversion membrane for interfacial heating. Due to the hydrophobicity, the PPy-coated SS mesh stays spontaneously at the water-air interface and converts light energy to heat, locally heating the surface water. (b) SEM images of the original smooth SS mesh surface (b1), and the PPy-coated mesh surface with microstructures (b2). Insets in (b1) and (b2) show the magnified view of the knitting wire surfaces. (c) Dependence of the thickness of the PPy coating on the electropolymerization time. (d) Water contact angles of the PPy-coated SS meshes after the hydrophobic modification. Insets in (d) show the shapes of a water droplet on the membrane at different tilt angles of $0^{\circ}$ (contact angle about $140^{\circ}$ ), $90^{\circ}$ and $180^{\circ}$, showing a typical Wenzel's wetting behavior.
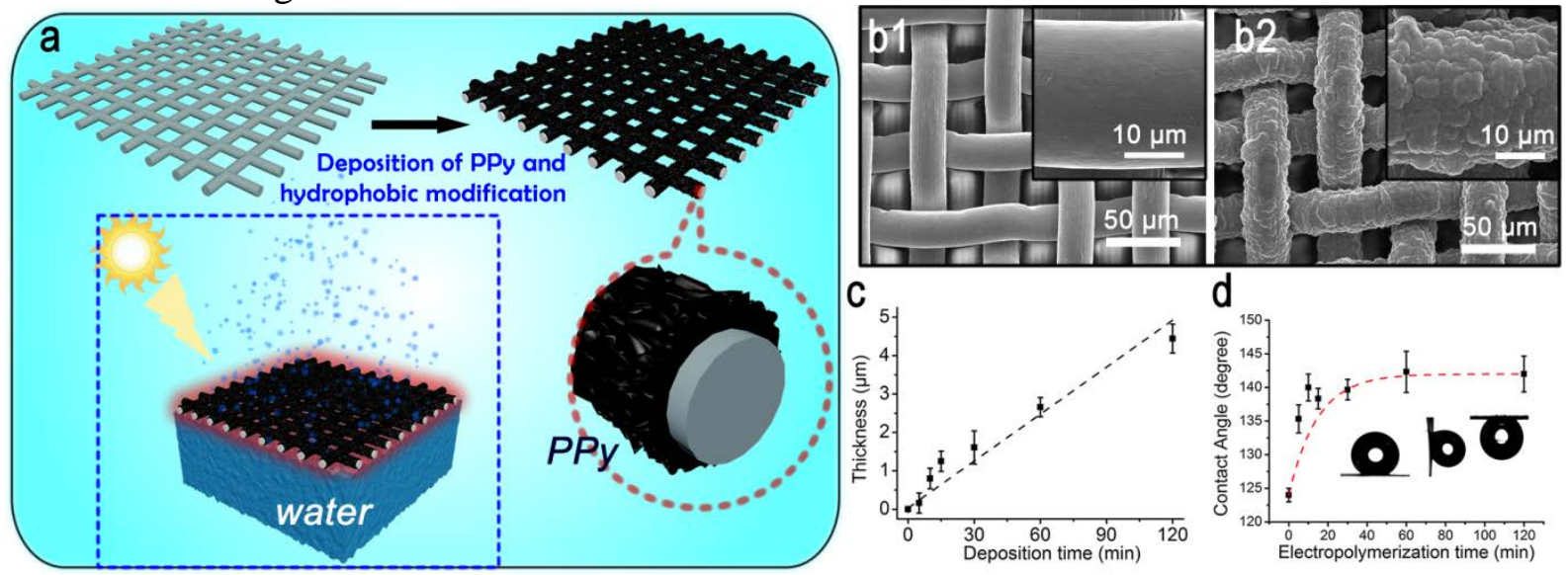


\section{WILEY-VCH}

Figure 2. Photothermal property of the PPy-coated SS mesh. (a) The steady-state temperatures of the PPy-coated SS meshes in air as a function of the thickness of the PPy coating, under the solar irradiation. (b) Time-course of the temperature of the original SS mesh and the PPy-coated (thickness $\sim 2.6 \mu \mathrm{m}$ ) SS mesh under the solar light irradiation. Red and black arrows indicate the time when the light was turned on and off for each sample, respectively. Insets in (b) show the IR thermal images of the PPy-coated versus original SS meshes under the solar light irradiation. The markers $(+)$ in the thermal images show the spots where time-course of the temperature curves were recorded.
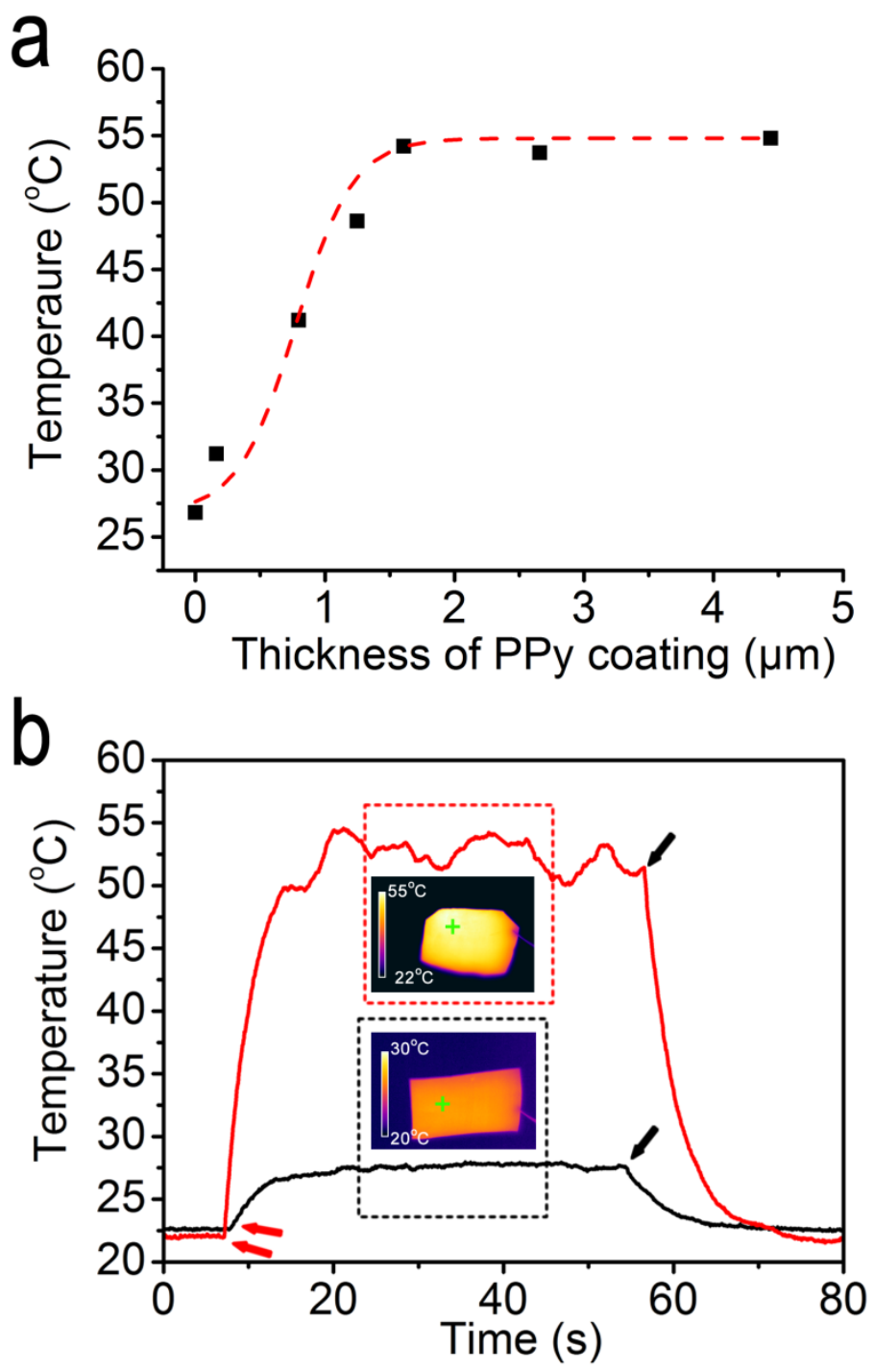


\section{WILEY-VCH}

Figure 3. Enhanced water evaporation by the hydrophobic photothermal membrane. (a) Schematic illustration for the setup of the water evaporation measurement with the PPycoated SS mesh floating on the water surface. Inset in (a) shows the digital photograph of the mesh floating on the water surface in a beaker. (b) Time course of water evaporation performance under various conditions. Black: water without light irradiation. Red: water under the light irradiation. Blue: water with an original SS mesh with hydrophobic modification. Pink, green, purple, and brown: water with floating SS meshes of different PPy coating thicknesses. (c) The water evaporation as a function of the thickness of the deposited PPy coatings on the SS mesh for 10 hours. (d) IR thermal images of water beakers without light (left panel), with light irradiation on for 20 seconds (middle panel) and for 2 hours (right panel). Left beaker: water only. Right beaker: water with the floating PPy-coated mesh on surface. (e) Temperature profiles of the marked lines in right panel of (d). (f) The surface temperature of the water with ( $\boldsymbol{\square}$, right beaker) and without ( $\square$, left beaker) the PPy-coated mesh under the solar light irradiation.

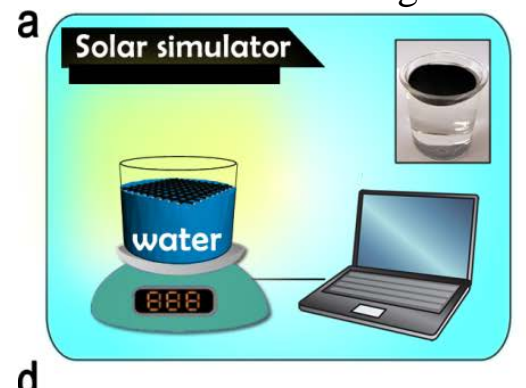

d

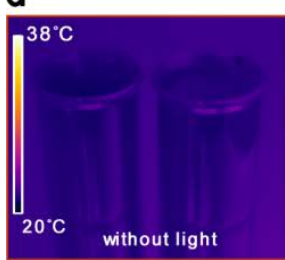

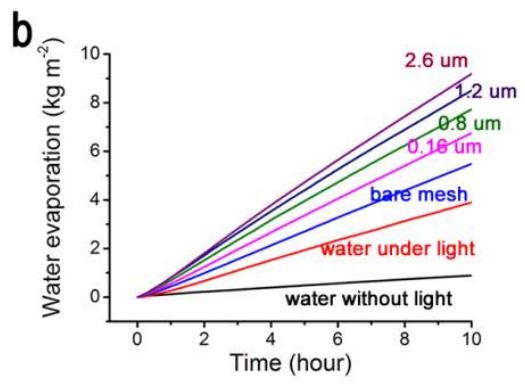

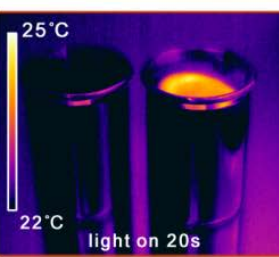

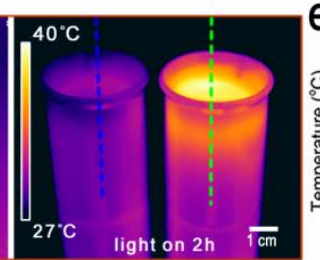

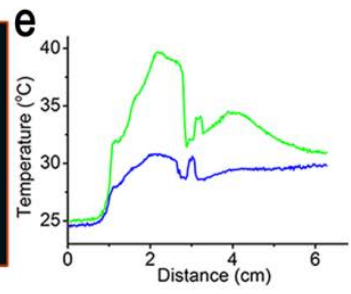

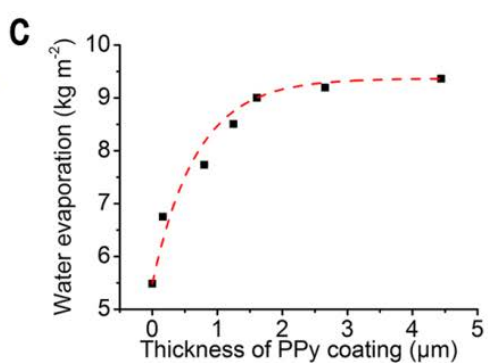

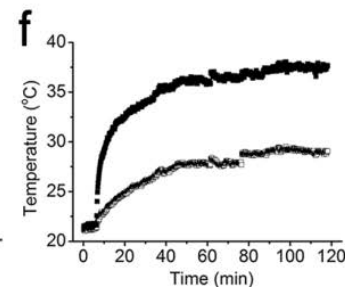




\section{WILEY-VCH}

Figure 4. (a) Contact angles of water droplets on the plasma treated PPy-coated mesh along with the healing timecourse at ambient conditions ( $\mathbf{\square}$ ) and under simulated solar light irradiation ( $\square$ ). (b) Change of water contact angles on the plasma-treated and light-irradiated (1 hour) PPy-coated mesh for 5 cycles. The insets in (b) were the shapes of the water droplets on the surfaces during one typical treatment cycle.
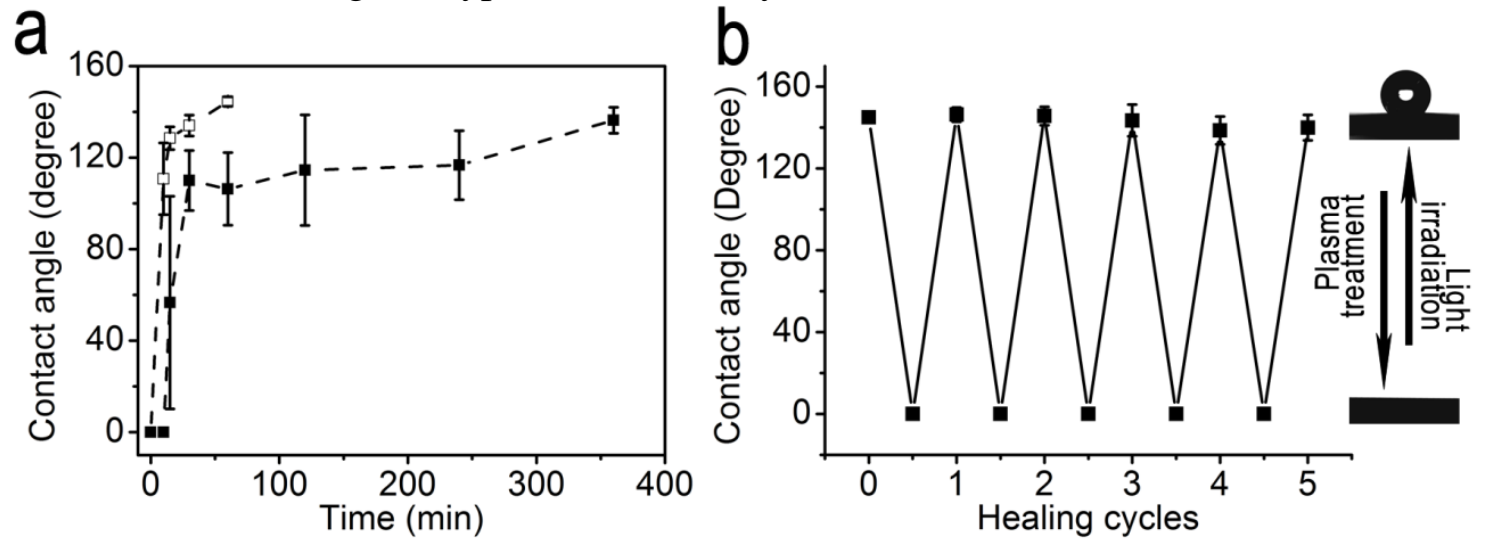


\section{WILEY-VCH}

Figure 5. Schematic configuration of the point-of-use device for direct and all-in-one solar distillation for freshwater production.

$$
\text { Point-of-use solar distillation device }
$$

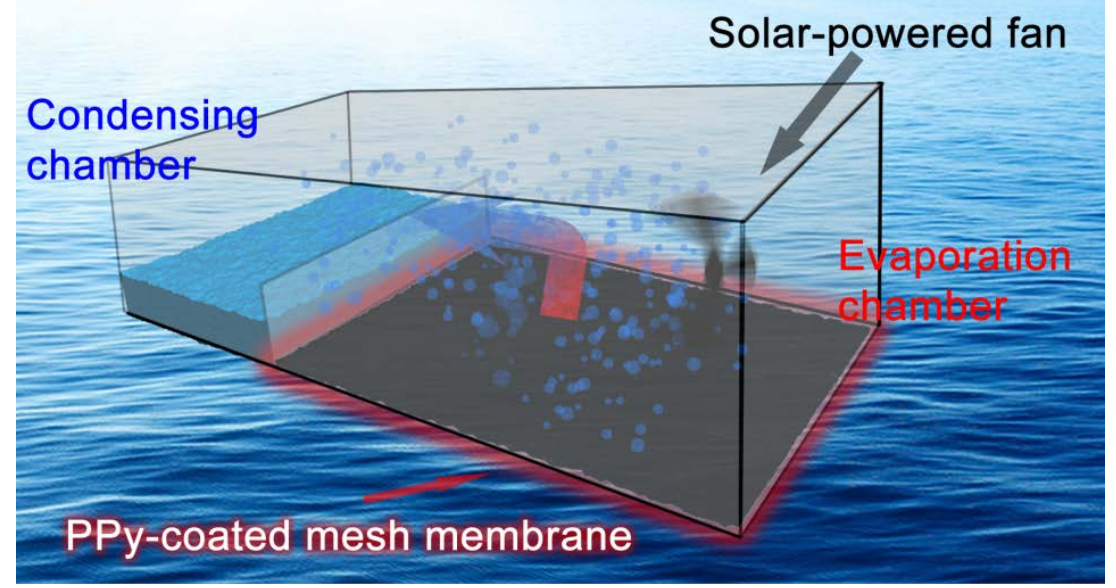




\section{WILEY-VCH}

Self-Healing Hydrophobic light-to-heat conversion membranes for interfacial solar heating are fabricated by deposition of light-to-heat conversion material of polypyrrole onto porous stainless steel mesh, followed by hydrophobic fluoroalkylsilane modification. The mesh-based membranes spontaneously stays at the water-air interface, collects and converts solar light into heat, and locally heats only the water surface for an enhanced evaporation. PPy: polypyrrole.

Keyword: light-to-heat conversion, self-healing, wetting, interfacial heating, membrane.

Dr. L. B. Zhang, B. Tang, Dr. J. B. Wu, R.Y. Li, Prof. P. Wang*

Hydrophobic Light-to-Heat Conversion Membranes with Self-Healing Ability for Interfacial Solar Heating

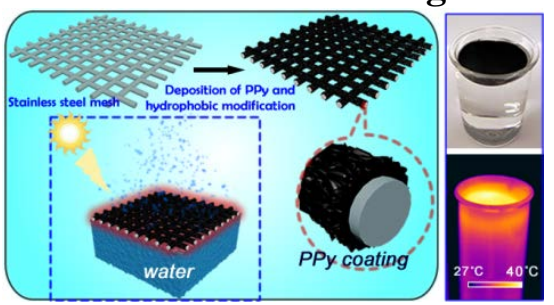

